\title{
El espacio social de los deshabitados en el Alto Aragón, España. Aproximación etnográfica a las actuales prácticas de ocupación como factor de conservación de un entorno
}

rural de alto valor paisajístico*

Cómo citar este artículo: Ruíz-Varona, A. (2018). El espacio social de los deshabitados en el Alto Aragón, España. Aproximación etnográfica a las actuales prácticas de ocupación como factor de conservación de un entorno rural de alto valor paisajístico. Cuadernos de Desarrollo Rural, ${ }_{15}$ (82), I-18. https://www.doi.org/10.III44/Javeriana.cdri5-82.edaa

Ana Ruíz-Varona

Universidad de San Jorge, España

nruiz@usj.es

ORCID: http://orcid.org/0000-000I-8807-4917

DOI: https://doi.org/ı.III44/Javeriana.cdrı5-82.edaa

Redalyc: http://www.redalyc.org/articulo.oa?id=II75772400I

Recibido: Io Noviembre 20I7 I Publicación: I8 Diciembre 2018

\section{Resumen:}

El actual mal estado de conservación de los deshabitados del Prepirineo oscense representa un alarmante escenario de pérdida de valores culturales, paisajísticos, arquitectónicos y sociales asociados a este territorio. El estudio cualitativo de las prácticas de su reocupación proporciona conocimiento sobre la motivación, por parte de unas minorías, por recuperar estos escenarios de vida. Los resultados determinan cuál ha sido el papel del medio natural a la hora de ocupar determinados deshabitados y si estas experiencias pueden llegar a constituir opciones de autonomía y viabilidad para su preservación, incluso en el marco de los actuales instrumentos de planificación espacial.

Palabras clave: patrimonio, paisaje, contraurbanización, etnografía. 


\title{
The Social Space of the Uninhabited Lands in Alto Aragón, Spain. Ethnographic Approach to the Current Occupation Practices as a Factor to Preserve a Rural Environment of Great Landscaping Value
}

\begin{abstract}
:
The current poor preservation conditions of the uninhabited lands in the Huesca Pre-Pyrenees represents an alarming scenery involving the loss of cultural, landscape, architectonic, and social values associated with this territory. The qualitative study of the reoccupation practices provides insights into the motivations by some minority groups to recover these life settings. The results thereof indicate the role of the natural environment when it comes to settle specific uninhabited lands and whether these experiences would eventually become an option for the land autonomy and a suitable option for preservation, even so under the framework of the current space planning provisions.
\end{abstract}

Keywords: patrimony, landscape, urban counter-development, ethnography.

\section{Introducción}

El punto de partida que toma el presente trabajo se centra en dos premisas relacionadas entre sí: en primer lugar, la creciente pérdida de actividad de las áreas rurales o de pequeño tamaño poblacional que inició en torno al segundo y tercer tercio del pasado siglo XX; y, en segundo lugar, el desajuste exponencial entre la variación de población y el territorio urbanizado experimentado desde esas fechas hasta la actualidad.

El problema que representan estos dos fenómenos en simultaneidad ha sido objeto de estudio y de propuestas de acción a nivel internacional (Halliday y Coombes, 1995; Martínez-Fernández, Kubo, Noya y Weyman, 20I2; Parlamento Europeo, r.o de junio de 20I6). España también cuenta con estudios a escala nacional (Arango, 1976; Collantes, 200I; Germán, 1995; López, Abellán y Godenau, 2009; Silvestre, 2003) y regional (Aguilar, 2009; AnautBravo, 2009; Andrey, 2009; Comas-D’Argemir, 1995). El interés común de todas estas referencias se basa no solo en aportar claves para entender mejor las causas y los efectos de este fenómeno en el territorio, sino también en incardinar a la propia disciplina urbanística y de ordenación del territorio en la definición metodológica y política para la mejora de tales ámbitos espaciales. De las investigaciones centradas en Aragón (Ayuda, Pinilla y Sáez, 2002; Slomp, 2004), merece especial atención el Centro de Estudios sobre la Despoblación y Desarrollo de Áreas Rurales (Ceddar), que ha venido realizando, desde el año 2000, una labor de estudio sobre las causas y consecuencias que explican el fenómeno - no solo en el ámbito de Aragón, pero sí de manera fundamental- de la despoblación (Sáez, Pinilla y Ayuda, 2001; Sanz, 2016) y sobre las políticas o acciones que han sido diseñadas para fomentar la reactivación demográfica de las áreas más deprimidas (Sáez, Ayuda y Pinilla, 2008), así como para preservar su patrimonio cultural y arquitectónico (Marín-Yaseli, 2006; Pujadas, I995; Sáez, 20I6; Sánchez, 1995). El centro se encarga de la edición de trabajos, monografías y de una publicación especializada de carácter anual (Ager: Revista de Estudios sobre Despoblación y Desarrollo Rural).

Ahora bien, si esta relación entre premisas se lleva al extremo, el escenario resultante de zonas escasamente pobladas y severamente despobladas pasa a caracterizarse por la existencia de núcleos deshabitados cuya conservación, en referencia a sus valores naturales, arquitectónicos, culturales, sociales y paisajísticos, queda amenazada.

Para el caso de Aragón, se observa que los instrumentos actuales de planificación espacial no han terminado de incorporar técnicas adecuadas para dar solución a la protección y mejora del estado de conservación (Gobierno de 
Aragón, 2005). A ello se suma que las actuales líneas de desarrollo territorial no han sabido posicionarse de manera efectiva a la hora de priorizar recursos para la conservación de los deshabitados, sobre todo cuando existen muchas áreas escasamente pobladas que luchan por su supervivencia (Gobierno de Aragón, 20r4).

Aun asumido este hecho, el interés que ofrecen los deshabitados es innegable, y su estudio, necesario (Acín y Pinilla, 1995). Los deshabitados constituyen una valiosa fuente de conocimiento de la arquitectura rural tradicional y de formas de vida pasadas. Este texto se centra, precisamente, en darle valor a la experiencia de aquellos agentes que durante estos últimos años han pretendido la recuperación de los deshabitados desde diferentes perspectivas, de manera que puedan inferirse acciones efectivas para integrarlos en los procesos de planificación urbanística y territorial que aborden su conservación y mejora.

Los estudios realizados, que, en cierto modo, convergen en este planteamiento de tomar experiencias de recuperación como variables de contexto que caracterizan a los deshabitados, se centran de manera general en una descripción exhaustiva de la morfología de lo construido, de su uso y del estado en que se encuentra en relación con las acciones emprendidas, aunque la mirada positiva muchas veces difiera de la realidad del problema a escala regional (Marín-Gavín, 20I8).

Sin embargo, el presente trabajo tiene como objetivo general, que da sentido a la investigación, conocer cuál ha sido el motor de cambio y las razones que justifican el empeño por parte de unas minorías de volver a activar determinados escenarios de vida ahora deshabitados. En esencia, se trata de estudiar si esta implicación responde a un interés social que pueda ser mantenido en el tiempo. El estudio pormenorizado de estas experiencias permite conocer cuál es el papel del medio natural a la hora de seleccionar determinados deshabitados frente a otros como posibles áreas de recuperación, además de saber si su ocupación responde a determinados patrones de localización a nivel regional o si estas propias experiencias, o su intensificación, pueden suponer un apoyo, o una amenaza, para la conservación y protección de este entorno rural de alto valor paisajístico.

Este trabajo considera que la contraurbanización, fenómeno de alteración en el modelo de poblamiento urbano entendido como búsqueda de una mejor calidad de vida, es una de las variables clave a la hora de estudiar las posibilidades que las experiencias de intervención social ofrecen en las áreas deshabitadas para su efectiva conservación, protección y defensa. Cloke (1985, 1988) explica la contraurbanización de las áreas remotas no solo a escala global -fundando su estudio en los cambios experimentados por la sociedad postindustrial-, sino a escala local, en la que centra la atención en los factores atractores de nuevos habitantes, como lo son el mercado del suelo y la calidad ambiental y natural del entorno de los deshabitados. A este respecto, se ha demostrado que las preocupaciones estructurales de mayor alcance influyen en la definición real de las motivaciones individuales por una búsqueda de un determinado estilo de vida (Benson y O’Reilly, 2009). Existe un número hasta la fecha creciente de personas y comunidades que se están desplazando a un medio rural en declive o abandonado (Elshof, Haartsen, van Wissen y Mulder, 2017), con lo que apuestan a la construcción social de una nueva ruralidad como escape de los males de la modernidad y la ciudad, así como por la conformación de unas redes de apoyo fundadas en las experiencias de la vida cotidiana y de subsistencia más básica, de praxis social. Este hecho condensa una visión alternativa al drama humano del desarraigo, derrotista y pasivo, que supone el incuestionable desequilibrio en la ocupación y administración del territorio, vinculado a los cambios políticos, sociales, culturales y económicos que han ocurrido desde la mitad de siglo XX (Mitchell, 2004).

Profundizar en las narrativas de este colectivo, que apuesta por esta nueva ruralidad, supone estudiar si el valor paisajístico, la calidad ambiental y la singularidad de un medio natural representan un factor atractor de primer orden en términos de reocupación de entornos urbanizados abandonados durante un periodo más o menos dilatado en el tiempo (Abrams, Gosnell, Gill y Klepeis, 20I2; Chi y Marcouiller, 20I3). En cierto sentido, todavía queda por evaluar en qué medida la valoración del espacio natural y del patrimonio cultural y construido está implicada en la valoración positiva de estas experiencias de ocupación de los deshabitados, determinando la propia estabilidad y viabilidad de estas experiencias en un marco temporal futuro. 
El presente trabajo pretende comprobar si el creciente número de experiencias de ocupación informal en el entorno de los deshabitados constituye una vía de autonomía para la gestión, el mantenimiento, la conservación y la protección de este entorno rural deshabitado. Para ello, se analiza una serie de datos de campo y se da explicación sobre las razones que llevan a reocupar los núcleos deshabitados y sobre el perfil tipo de población (si existe) más activo en este tipo de prácticas.

La consecución de este objetivo distingue una primera etapa del estudio que aborda la problemática en torno a la despoblación y el declive del medio rural para un ámbito espacial concreto que el trabajo toma como caso de estudio: la región del Alto Aragón, en España. En una segunda fase, se describe la metodología diseñada para la generación de las fuentes primarias de información analizadas. Los resultados obtenidos quedan referidos en el tercer apartado; la cuarta sección se dedica a la discusión de estos, y el quinto apartado, a las conclusiones y reflexiones finales extraídas del trabajo realizado.

\section{El Alto Aragón como caso de estudio: el paisaje natural y la herencia construida inactiva que se está perdiendo}

Conviene realizar de manera previa dos aclaraciones con respecto a los términos empleados en el presente trabajo. En primer lugar, y en general, la denominación Alto Aragón (figura I) hace referencia a la región septentrional de Aragón, correspondiente a la provincia de Huesca. No obstante, otras fuentes toman como referencia la delimitación dada hace varias décadas, correspondiente al área prepirenaica y pirenaica de la región. En correspondencia con esta segunda definición, aquí se toma como referencia la delimitación correspondiente a las cuatro comarcas pirenaicas: La Jacetania, Alto Gállego, Sobrarbe y La Ribagorza. En segundo lugar, a pesar de que no existe ninguna definición oficial, el término empleado para referirse a los lugares de estudio será deshabitados, en vez de abandonados; la característica fundamental es que allí no vive nadie (empadronado de manera oficial), independientemente del régimen de titularidad (con diferentes regímenes, pero no abandonado) y del grado de conservación (en este caso, el estado de abandonado se considera subjetivo).

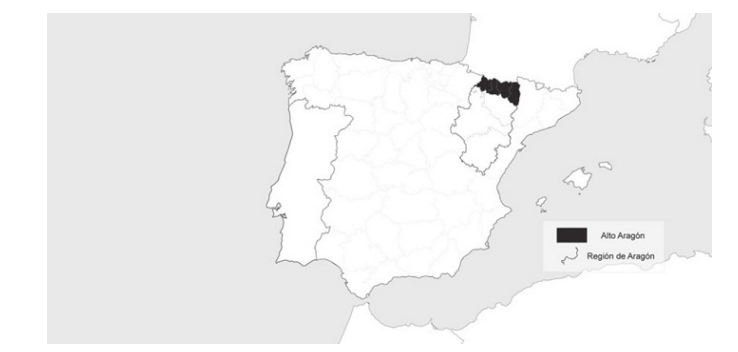

FIGURA 1

Localización del Alto Aragón en la región de Aragón, España

FUENTE: ELABORACIÓN PROPIA

En la región del Alto Aragón existe un número elevado de núcleos deshabitados en un medio natural de alto valor paisajístico. Su estudio se ha centrado casi de manera esencial en documentar su estado actual (Acín, 1994, 1997; Acín y Pinilla, 1995; Bernard-Rivera y Castellanos, 1982; Castán, 2009; Castán, Calavera e Iglesias, 1990; Díaz, 1995; Laglera, 20I0, 20I4a, 20I4b, 20I5; Satué, I99I; Serrano, 2007; Ubieto, 1984), en diseñar metodologías 
de trabajo con el objeto de estudiar su marco territorial y legislativo y en determinar su estado de conservación y titularidad (Marín-Gavín, 2013), así como -en menor medida- en recopilar información y diseñar posibles medidas de recuperación y reactivación de los pueblos (Aineto..., 1984; Bernard-Rivera, 1992; Laliena, 2004; Lardiés y Castelló, 2015; Presidencia de las Cortes de Aragón, 2000; VVAA, s. f.).

Según los estudios de Ayuda y Pinilla (2002), el derrumbamiento demográfico del Pirineo aragonés ha supuesto que, desde finales del siglo XIX hasta nuestros días, el conjunto de las comarcas pirenaicas aragonesas haya experimentado tasas de crecimiento demográfico negativas, por lo que se redujo la población a más de la mitad. Las causas las atribuyen tanto a una aguda crisis de su economía tradicional como a la atracción que genera sobre la población pirenaica la existencia de áreas próximas de oportunidad en términos económicos y productivos. El proceso de despoblación más intenso abarca desde 1940 hasta 1960 y queda inscrito en lo que ellos han determinado como los ritmos de descenso de población más altos, correspondientes a las décadas 1920-1970.

Los datos de deshabitados (figura 2) refuerzan la hipótesis de que este fenómeno ha afectado de manera distinta a gran parte del Alto Aragón, más allá de las situaciones coyunturales derivadas de la construcción de embalses o del proceso de reforestación. Las comarcas situadas entre los Pirineos y las sierras exteriores, es decir, las de La Jacetania, el Alto Gállego, Sobrarbe y La Ribagorza, albergan una cantidad alta de deshabitados, dentro de los que se distinguen enclaves esenciales como la Garcipollera, el Valle del Guarga o la zona del Sobrepuerto. Su número, aunque se advierten pequeñas variaciones según la fuente primaria, se estima en torno a los I92, si se consideran exclusivamente los núcleos de población ( Red Natural de Aragón, 2003-2007).

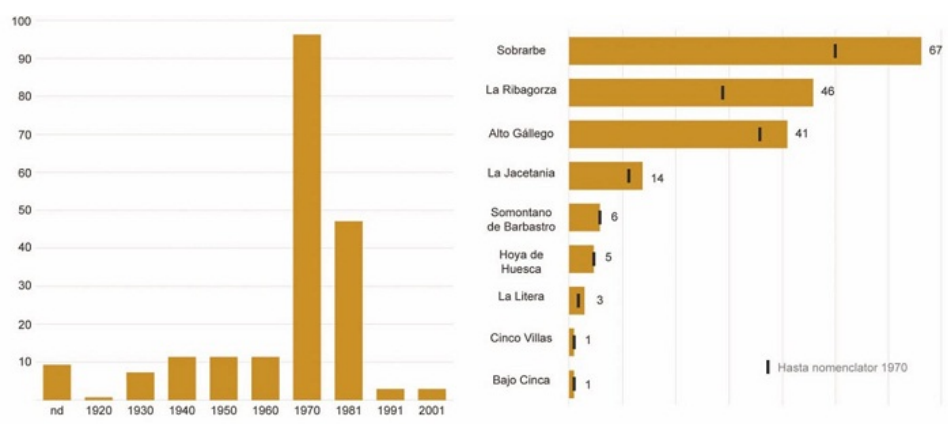

FIGURA 2

Número de deshabitados QUe deJAN de figurar EN El NOMENClátor desde 1920 hasta la aCtualidad; COMPARATIVA SEGÚN PERTENENCIA A COMARCAS HASTA 1970 Y HASTA LA ACTUALIDAD Fuente: elaboración propia con base en datos de NOMENCLÁtor y del Departamento de Medio Ambiente del Gobierno de Aragón

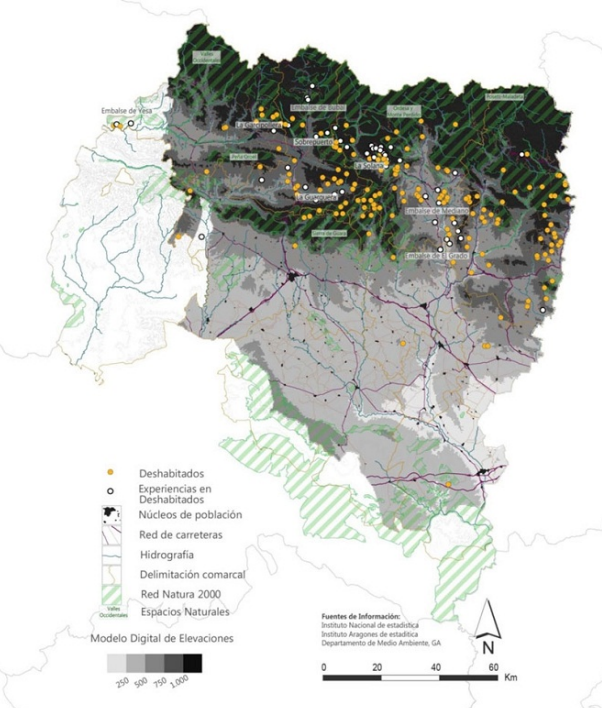

FIGURA 3

Contexto territorial en el que se localizan los deshabitados del Alto Aragón Fuente: elaboración propia con base en el Instituto Nacional de Estadística (INE), el Instituto Aragonés de Estadística y el Departamento de Medio Ambiente del Gobierno de Aragón 
Si la atención se centra de manera exclusiva en las entidades singulares de población, en 2015 es 3 I el número de pueblos deshabitados del Alto Aragón frente a los 68 del conjunto de la comunidad autónoma (figura 3). El análisis del modelo digital de elevaciones demuestra que el $80 \%$ de los deshabitados alcanza cotas superiores a los 1000 metros, correspondientes con la montaña pirenaica altoaragonesa. Este accidentado relieve, en combinación con las escasas comunicaciones por carretera con las demás zonas dinámicas, dibuja el contexto en el que surge el principal problema de la montaña pirenaica: la desarticulación de su economía tradicional, basada en la ganadería ovina trashumante, la producción agrícola para el autoconsumo y las actividades de transformación de algunas materias primas. Tal como afirman Ayuda y Pinilla (2002), "las dificultades crecientes para seguir desarrollando estas actividades en su forma tradicional condujeron a un auténtico proceso de desmantelamiento de las bases sobre las que se sustentaba la vida económica de la zona” (p. II4). Con ello, mucha de la arquitectura doméstica (rural) del Alto Aragón, ampliamente estudiada (Allanegui, 1979; Feduchi, 1976; Flores, 1973; Naval-Mas, 1988), se ve sometida a un proceso de abandono y degradación que, intensificado, se mantiene hasta nuestros días.

Sin embargo, y teniendo en cuenta el hecho de que sin mejores oportunidades en otras áreas próximas (Zaragoza y Barcelona, además de Jaca y Sabiñánigo como motores turísticos e industriales, respectivamente) no habría existido un proceso de despoblación de semejante proporción, merece la pena reseñar aquellas oportunidades productivas para las que el Pirineo todavía ofrece ventajas comparativas, como el despegue del turismo interior de montaña y su especialización, en concreto, desde el punto de vista medioambiental. El Alto Aragón comprende parajes de reconocida belleza y riqueza cultural que suponen aproximadamente el $9 \%$ del territorio; esta relación triplica, prácticamente, al resto de la comunidad autónoma (Gobierno de Aragón, 20II-2015). De manera particular, la atención se centra en los espacios naturales protegidos, como la peña Oroel, la sierra y los cañones de Guara, el valle de Ordesa o los ibones y las morrenas en torno a Posets y Maladeta. Tanto las zonas especiales de conservación (ZEC) como las zonas de especial protección para las aves (ZEPA), ambas integrantes de la Red Natura 2000, configuran una red de espacios conectados de reconocido interés medioambiental y patrimonial en torno a la que se localiza la mayoría de despoblados.

\section{La metodología etnográfica}

El procedimiento aplicado ha sido desarrollado a partir de una metodología de análisis etnográfica. Existen unos primeros trabajos etnográficos y geográficos que refieren al Alto Aragón (Allanegui y Souto, 200z; Briet y Acín, 2003; Caro-Baroja, 199I), sin embargo, todavía son escasas las referencias que abordan desde este punto de vista el estudio de las actuales prácticas de ocupación de los deshabitados, y los estudios precedentes se encuentran únicamente en el ámbito internacional (Benson, 20II; Dam, Heins y Elbersen, 2002).

Las fuentes consultadas en la investigación, aparte de las explicitadas en el texto, incluyen ortofotografías y planos provenientes de la Infraestructura de Datos Espaciales (Idearagón) del Instituto Geográfico de Aragón (Igear), el catálogo de pueblos y municipios de Aragón referido a la estadística de población y nomenclaturas toponómicas entre 1900 y 2004 (Gavín Lanzuela, 2005), información alfanumérica sobre demografía y población rescatada del Instituto Aragonés de Estadística (Iaest), artículos de prensa y archivos personales de antiguos residentes, comunidades e investigadores.

El trabajo se ha realizado considerando tres fases. La primera de ellas corresponde a una labor de recopilación y selección de inventarios acerca de la situación actual de los deshabitados en el Alto Aragón. Como criterio general, se revisa el contenido de la publicación "Huesca de la A a la Z" (Castán, 2009; Castán et al., 1990) y se complementa con los estudios realizados por Marín-Gavín (20I3), Acín (1994, 1997) y Laglera (2014a, 20I4b, 20I5). 
La segunda fase se centra en la selección de prácticas de ocupación de deshabitados en el Alto Aragón (figura 3). Se analiza el contenido de las monografías que recogen diversas experiencias de intervención en el Alto Aragón fundadas tanto en el levantamiento y análisis del estado actual del conjunto edificatorio frente a épocas pasadas como en los agentes que toman parte y en herramientas de planificación que actualmente afectan a tales ámbitos espaciales (Ruiz, 20I4). Se obtiene, así, una primera cartografía SIG (sistema de información geográfica) en sentido amplio que engloba una diversidad de prácticas de ocupación.

Por un lado, la tercera fase consiste en la inmersión etnográfica durante un periodo temporal corto; esta se realiza con el objetivo de identificar patrones de comportamiento y de cambio en el territorio asociados al estilo de vida actual y pasado, y por otro lado, la tercera fase incluye, como herramienta de análisis cualitativo, la realización de entrevistas semiestructuradas en profundidad con informantes clave y personas relacionadas de manera directa con las prácticas de ocupación de los deshabitados en el Alto Aragón, de tal manera que se seleccionaron aquellas experiencias que tienen una especial significancia con respecto al conjunto: un total de 15 experiencias, de las que se presentan 9 en detalle (tabla $\mathrm{I}$ ).

TABLA 1

INFORMACIÓN DE LOS ENTREVISTADOS

\begin{tabular}{|c|c|c|c|c|}
\hline \multirow[t]{2}{*}{ Prácticas de ocupación } & \multicolumn{4}{|c|}{ Entrevistado } \\
\hline & Género & $\begin{array}{l}\text { Eda } \\
\text { d }\end{array}$ & Procedencia & $\begin{array}{l}\text { Relación con el } \\
\text { deshabitado }\end{array}$ \\
\hline Deshabitado 1 & $\begin{array}{l}\text { Masculin } \\
\mathrm{o}\end{array}$ & $>50$ & UE & Habita \\
\hline Deshabitado 2 & $\begin{array}{l}\text { Masculin } \\
\mathrm{o}\end{array}$ & $>40$ & $\begin{array}{l}\text { Otra provincia de } \\
\text { España }\end{array}$ & Visita \\
\hline Deshabitado 3 & Femenino & $>40$ & Extranjero no UE & Habita \\
\hline Deshabitado 4 & $\begin{array}{l}\text { Masculin } \\
o\end{array}$ & $>40$ & $\begin{array}{l}\text { Otra provincia de } \\
\text { España }\end{array}$ & Habita \\
\hline Deshabitado 5 & Femenino & $>20$ & $\begin{array}{l}\text { Otra provincia de } \\
\text { España }\end{array}$ & Habita \\
\hline Deshabitado 6 & Femenino & $>30$ & UE & Habita \\
\hline Deshabitado 7 & $\begin{array}{l}\text { Masculin } \\
o\end{array}$ & $>50$ & Aragón & Trabaja \\
\hline Deshabitado 8 & $\begin{array}{l}\text { Masculin } \\
\mathrm{o}\end{array}$ & $>40$ & Aragón & Trabaja \\
\hline Deshabitado 9 & Femenino & $>40$ & Aragón & Trabaja \\
\hline
\end{tabular}

Fuente: elaboración propia

Dentro de este formato semiestructurado, se trataron de abordar, de manera flexible, tres temas: i) el análisis histórico, con objeto de conocer la época y la coyuntura en las que los pueblos fueron deshabitados, prestando especial atención al grado de conocimiento que de ello tenían las personas y comunidades, así como las circunstancias e intereses que motivan su ocupación; 2) el modo de actuación diseñado y llevado a cabo, y 3 ) su valoración de la experiencia y viabilidad futura. Adicionalmente, se realizó un taller de análisis con alumnos universitarios que contó con la participación de técnicos del servicio de estrategias territoriales de la administración regional del Gobierno de Aragón y que supuso un aporte de conocimiento colectivo para explorar, a través de los resultados, una situación que es difícil de abordar desde solo una plataforma de trabajo. 
En ocasiones, los nombres propios, fundamentalmente con respecto a las personas que amablemente accedieron a conversar con el equipo de trabajo y a los pueblos que actualmente ocupan, han sido preservados en el anonimato, por lo que en el texto aparecen con seudónimos por petición explícita y razones obvias.

\section{Resultados: prácticas de ocupación de los deshabitados en el Alto Aragón}

En términos generales, los 9 estudios de caso seleccionados (tabla I) fueron deshabitados en la década de los 60 y 70 del siglo XX por motivos de reforestación, construcción de presas y embalses o desplazamiento de la población a núcleos aledaños más dinámicos. Todos ellos poseían construcciones medievales de interés, y muchas de estas contaban con pinturas murales populares.

Uno de los aspectos considerados que ha dotado de mayor contenido a la investigación ha sido el modo como los individuos ocupan los deshabitados y ejercen sus prácticas de vida cotidiana, ya que, a pesar de las circunstancias variadas que los han llevado a interesarse en un primer momento en estos lugares, en muchos casos el factor determinante de movilización ha sido el entorno natural y el paisaje de montaña en los que tienen lugar estas prácticas. En ese sentido, es paradigmático el ejemplo de Ellen Sewall, joven licenciada en periodismo y antigua residente en Barcelona:

Me interesé por los modos de vida alternativa gracias a la influencia que tuvo un profesor a comienzos de carrera [Arcadio Oliveres, activista, licenciado en ciencias económicas y defensor del decrecimiento]; tras conocer más, empecé a experimentar esta nueva vida y ya llevo seis años aquí [...]. Una de las diferencias más notables es que mi vida cotidiana ha cambiado de manera radical. (E. Sewall, comunicación personal, ir de mayo de 2016)

Tanto Ellen como el resto de personas que viven en este entorno han tomado la decisión de aprender a vivir con unos medios muy básicos, en un contacto permanente con la naturaleza y con los recursos que esta proporciona.

La vida en proximidad con el entorno natural parece tener relación con el modo de vida alternativo demandado tras las dificultades encontradas en la ciudad, fundamentalmente en periodos de crisis laboral y económica. Lupe completó los estudios universitarios y convive con otros licenciados en magisterio, educación social y derecho o con ingenieros forestales. Para ellos, existe un alto número de personas que, tras finalizar la carrera, se encuentra con una salida profesional árida y artificial que, en sus palabras, "te somete a vivir de una manera que ni deseas ni esperabas [...], así que buscas una alternativa al vacío que sientes en tu vida, porque si no, no aguantas” (Lupe, comunicación personal, in de mayo de 20I6).

Si bien la media de edad que caracteriza a toda la comunidad no supera la treintena, existen casos de personas con mayor edad, como el de Jepe, un holandés que lleva viviendo en este deshabitado desde hace un año. Como explica él,

la principal razón para desplazarme hasta aquí, sin conocer a nadie, fue precisamente la confianza nula en el futuro que te espera en tu lugar de origen. En mi caso, el contexto de crisis económica de mi país me imposibilitaba cierta garantía de estabilidad con respecto a la tenencia o alquiler de una vivienda. Iba a estar sometido de por vida. (Jepe, comunicación personal, 23 de marzo de 2016)

En el caso de Jepe, la búsqueda de soluciones a su situación de incertidumbre la realizó a través de internet; le interesaron los pueblos de montaña en España que han sido abandonados en décadas pasadas, y por eso señala que estos pueblos son muy conocidos en determinados círculos de su país y tienen mucho atractivo. Él encontró una pequeña comunidad que promociona, a través de una red social, una vida en comunidad autosostenible y decidió 
explotar esta opción. Llegó solo, como también lo hizo Shane, un escocés que se siente afortunado por poder vivir en este lugar de montaña y de espectacular paisaje, puesto que es difícil encontrar un entorno de montaña con estas condiciones en su propio país, que esté en la naturaleza y que tenga tantos días de sol.

Resulta revelador que todas las personas que provienen de un país extranjero, preeminentemente del norte de Europa, confirman que una de las razones fundamentales por las que se van a vivir a deshabitados es el hecho de que se pueda vivir en la naturaleza, alejadas de las dinámicas urbanas que más difieren en términos de tiempos de desplazamiento, ruidos y paisaje urbano.

El tiempo de permanencia en estos deshabitados es indeterminado para ellos. En determinados casos, existe un compromiso previo por querer vivir allí de manera permanente, con lo que evitan la temporalidad y cualquier alusión a una segunda vivienda o lugar de vacaciones, como es el caso de Mipanas. La primera vez que Sadhu descubrió Mipanas fue gracias a su afición por escalar, hace ya casi treinta años; el paraje era excepcional y decidió quedarse allí y dejar su trabajo en Barcelona. Ahora, ya han pasado dos generaciones y viven is personas, todas ellas con estrechos lazos familiares o con el convencimiento de querer un modo de vida alternativo al sometimiento que se tiene en la ciudad. Esta realidad corresponde a un nivel primario de organización en comunidad, que establece una vinculación entre individuos de manera íntima, directa y personal, sin que esta tenga que responder a intereses por un objetivo concreto o tenga que estar forjada en la pertenencia a una determinada posición social. En realidad, "si alguien quiere ir a vivir a este lugar tiene que tener claro que esto no es un pueblo de vacaciones, aquí queremos gente que viva todos los días, que se relacione y seamos vecinos" (Sadhu, comunicación personal, 20 de julio de 2016).

Sin embargo, también existen experiencias que se caracterizan por ser precisamente eso, un lugar de vacaciones o de visitas ocasionales donde el contacto permanente con la naturaleza también está presente. En este caso, la ocupación se realiza por parte de propietarios privados o de fundaciones o asociaciones en deshabitados.

El caso de Isín o Ligüerre de Cinca responde a los intereses de fundaciones o asociaciones por desarrollar su actividad en alguno de estos deshabitados. Los responsables del desarrollo de estos proyectos recuerdan que lo más difícil fue garantizar la financiación para llevar a cabo el proyecto, además de

buscar un pueblo adecuado para nuestro proyecto. Inicialmente, pensamos en algún pueblo como Lanuza o Mipanas, pero existía siempre la dificultad de que alguna parte sí que estaba ocupada y no fue posible que nos pudieran ceder el pueblo [...]. El régimen de titularidad es el de cesión por 50 años, prorrogable otros tantos años más. (A. Rufas, comunicación personal, i6 de marzo de 2016)

En este caso, la oportunidad de ocupar un deshabitado es resultado de una evaluación previa, en la que se valoran aspectos de régimen de propiedad, movilidad, funcionalidad a escala comarcal y potencial de desarrollo turístico, no solo teniendo en cuenta la proximidad del Pirineo, sino también de embalses y cursos de ríos. "Centro de vacaciones" o "Un pueblo para ti" son dos de los lemas que se han diseñado y promocionado para atraer a usuarios temporales. Es por ello que la cobertura de una infraestructura adecuada al uso es fundamental, tal como revela la encargada de uno de estos centros de vacaciones; también lo es la promoción por redes sociales y páginas web de alojamiento online, ya que tienen su máxima demanda por parte de turistas franceses, ingleses, holandeses y, en menor medida, alemanes. Su experiencia les hace asegurar que este tipo de intervenciones, cuyo acondicionamiento y puesta a punto rondan los 25 años de duración, deben estar apoyadas por la administración para que puedan llevarse a cabo con ciertas garantías de éxito. José Antonio, director de otro de los centros, manifiesta que

no es fácil acometer un proyecto de este tipo, porque existe un gran vacío con respecto a cómo recuperar los deshabitados, y tan solo son rentables aquellos en los que el turismo sufraga los gastos de mantenimiento y de gestión. (A. Rufas, comunicación personal, i6 de marzo de 2016)

También existen experiencias de particulares que, de manera complementaria a esta búsqueda de vida alternativa, quieren hacer de los deshabitados un lugar de encuentro ocasional y vacacional. El holandés Jacobo Scheltus se interesó por la historia de un núcleo deshabitado que hace unos años compró, pero declara que "el 
proceso no fue fácil, sobre todo en lo que respecta a poner de acuerdo a los pocos propietarios de las casas medio en ruinas” (J. Scheltus, comunicación personal, 2 de marzo de 20I6).

Ahora, Scheltus convive en Sierra Estronad con su mujer e hijo, de forma austera, y promociona su modo de vida y la posibilidad de formar parte de él por un periodo determinado por medio de las tecnologías de la información y la comunicación (TIC). La red informática se ha convertido en la herramienta perfecta para dar a conocer el paraje natural en el que viven y para ofertar hospedaje indefinido a visitantes a cambio de su ayuda y trabajo en tareas de reconstrucción, horticultura o conservas, según la temporada. Hasta el momento, su oferta ha seducido a personas de diversas procedencias (Dinamarca, Estados Unidos, Francia, Suiza e Israel) y con objetivos muy variados, que comprenden desde la redacción de un trabajo de investigación (búsqueda de aislamiento) hasta el intercambio cultural como enriquecimiento personal (interés por residir en un entorno próximo de montaña alejado de las principales urbes) o la aplicación práctica de técnicas de construcción en un entorno rural (campo de trabajo). Aunque su régimen de permanencia es estacional, la llegada de nuevos ocupantes -su rotación- permite que esta experiencia, en términos generales, tenga visos de mantenerse constante por un periodo de tiempo mayor.

El objetivo de Jacobo ha sido desde el inicio la reconstrucción de los despoblados de manera fidedigna a como lo documentan los textos y planos existentes del área, que ha ido adquiriendo y revisando en detalle. Este es precisamente el factor diferencial que tratan de promocionar a través de diferentes plataformas web (disponen de internet por emisión de radio desde hace unos años). Los terrenos cuentan con un emplazamiento privilegiado en las estribaciones del Pirineo oscense. El entorno sobre el que se desarrolla la cotidianidad de los Scheltus cuenta con numerosos elementos de calidad paisajística: amplias faldas de montaña cubiertas por hectáreas de bosque virgen en una primera fase y, como fondo, los mallos y cañones que perfilan el Parque Cultural de San Juan de la Peña al norte. Además de la ausencia de ruidos y de la presencia de la fauna y flora autóctona, se suman los campos de cultivo de cebada y avena y los muros de piedra, que dan un contexto de recogimiento y sosiego al conjunto de edificaciones de piedra que se organizan en torno a una iglesia, en la mayoría de los casos en un estado muy degradado, y que quieren restaurar. Su hijo se ha formado en el oficio de cantero y aplica las técnicas tradicionales de construcción a las edificaciones sobre las que han intervenido hasta ahora. La meticulosidad con la que se enfrentan los Scheltus a las tareas de reconstrucción o de acondicionamiento de los edificios, que en general se encuentran en estado de ruina, es muy difícil de encontrar en este tipo de experiencias, pero tampoco es garantía suficiente de un buen resultado.

La familia de Sadhu también ha intervenido sobre la edificación, incluyendo operaciones de aumento de planta, aperturas de hueco o miradores. Ellos conviven con tres familias más en un régimen de cooperativa en Mipanas, tras haber conseguido una cesión del deshabitado por parte del propietario, que en este caso era un organismo público. La solución final ha sido cuidada, pese a que en ocasiones se alejan de las técnicas tradicionales de construcción. Ellos mismos son conscientes de que a día de hoy no existe ningún documento específico que guíe las posibles reformas de las construcciones, así como de los recursos limitados que individualmente destinan a esta labor (figura 4 ).
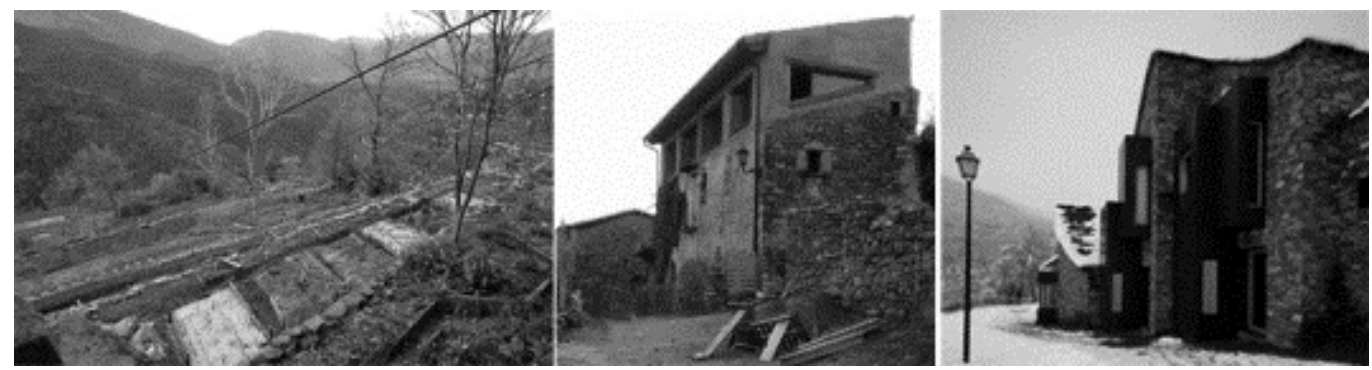

FIGURA 4

Huertos con técnica de abono verde y construcciones reformadas en Mipanas y en Isín

FuENTE: ELABORACIÓN PROPIA (LOS HUERTOS CON TÉCNICA DE ABONO VERDE Y LAS CONSTRUCCIONES REFORMADAS EN Mipanas); WWW.isin.es (CONSTRUCCIONES Reformadas En Isín) 
Muchos de los deshabitados se encuentran en emplazamientos remotos sin apenas vías de acceso, ninguna infraestructura activa de electrificación o canalización de aguas ni servicios básicos como correos, asistencia sanitaria o educativa. Precisamente, la dependencia de los pequeños núcleos de población activos del entorno más próximo permite la estancia más o menos continuada en los deshabitados. Sadhu se desplaza diariamente a los núcleos de población aledaños, donde trabaja como autónomo en actividades de construcción especializada. De cierta manera, esta vida alternativa nace de las demandas de un sector de la población inserto en las lógicas de la sociedad del consumo y de las que pueden valerse para promocionar el atractivo de los deshabitados.

En ocasiones, se han diseñado acciones para contrarrestar esta situación de necesaria demanda de desplazamiento a los núcleos de población más próximos, aunque los recursos son muy limitados y la dependencia es innegable. Ellen trabaja en la huerta a través de técnicas de abono verde, cultivando hortalizas y verduras como principal recurso para alcanzar cierto grado de autosuficiencia. También realiza pequeños trabajos de costura y elabora ungüentos naturales que intercambia por otros productos en encuentros rurales y con población de áreas vecinas, con lo que ha consolidado una red informal de proximidad que da soporte a sus necesidades más básicas. Sin embargo, afirma Ellen, "es necesario al menos comprar alimentos básicos como arroz o leche al menos cada dos semanas, por lo que tenemos que disponer de algún coche para llegar al supermercado más cercano” (E. Sewall, comunicación personal, ir de mayo de 20I6).

Los asuntos médicos y las gestiones administrativas básicas los sigue realizando en su ciudad de origen, lo que supone abandonar su vida alternativa por unos días. Ella misma admite que no existe ningún tipo de ayuda ni motivación para vivir en estas áreas más que la propia convicción de querer una vida aislada y tranquila, cuestión que le hace entender su experiencia en los deshabitados como algo -indefinidamente- estacional. Ellen reconoce que el teléfono móvil e internet son muy útiles, sobre todo como recurso que alivia las inseguridades de dejar a la familia y los amigos o que mantiene un sentido de lo familiar en un entorno desconocido. Pese a que ya lleva varios años okupando una pequeña borda en el monte, el sentido de continuidad con respecto a su vida anterior fundado en el uso de las TIC- le da la sensación de poder regresar a ella en cualquier momento.

\section{Discusión}

A partir del análisis contrastado se comprueba la existencia de tres categorías en las prácticas de ocupación relativamente homogéneas.

En primer lugar, se identifican pueblos deshabitados ocupados por los antiguos propietarios o comprados a posteriori por un particular o un conjunto de particulares con el objetivo de mantener el patrimonio construido, preservarlo y rehabilitarlo. No es una práctica mayoritaria y muchas veces responde a sentimientos de nostalgia, y las pocas experiencias encontradas revelan grandes dificultades para su realización.

En segundo lugar, se identifica un número algo mayor de deshabitados ocupados de manera regular en régimen de cesión u okupados. El objetivo perseguido por estas personas se centra en alcanzar una vida alternativa en comunidad y en contacto con la naturaleza, y en ocasiones intervienen en el patrimonio heredado con el objeto de mantenerlo en uso y reactivarlo, de acuerdo con las ideas de economía común y de desarrollo social y ecológico sostenible.

En tercer y último lugar, los pueblos deshabitados también son objeto de intervención, a propósito del desarrollo turístico especializado, por parte de diferentes asociaciones que son apoyadas en mayor o menor medida por la administración y que, en cualquier caso, guían su intervención desde el supuesto de recuperar los valores tradicionales del lugar (tabla 2). 
TABLA 2

Categorías de prácticas de ocupación en el Alto Aragón

\begin{tabular}{|c|c|c|c|}
\hline $\begin{array}{l}\text { Prácticas de } \\
\text { ocupación }\end{array}$ & $\begin{array}{l}\text { Conocimiento y } \\
\text { motivación }\end{array}$ & Modo de actuación & $\begin{array}{l}\text { Valoración y } \\
\text { viabilidad }\end{array}$ \\
\hline \multicolumn{4}{|c|}{ Uso particular y puesta en valor } \\
\hline Práctica 1 & Alto & Propietario (subvenciones, capital privado) & A largo plazo \\
\hline Práctica 2 & Medio & Propietario (capital privado) & Incierta \\
\hline Práctica 3 & Alto & Propietario (subvenciones, capital privado) & Incierta \\
\hline \multicolumn{4}{|c|}{ Okupación y vida alternativa } \\
\hline Práctica 4 & Medio & Libre (subvenciones, capital privado) & $\begin{array}{l}\text { A mediano } \\
\text { plazo }\end{array}$ \\
\hline Práctica 5 & Alto & Libre & A corto plazo \\
\hline Práctica 6 & Medio & Libre & $\begin{array}{l}\text { A mediano } \\
\text { plazo }\end{array}$ \\
\hline \multicolumn{4}{|c|}{ Recuperación y desarrollo turístico especializado } \\
\hline Práctica 7 & Medio & $\begin{array}{l}\text { Empresarial (subvenciones, convenios, } \\
\text { capital privado) }\end{array}$ & $\begin{array}{l}\text { A mediano } \\
\text { plazo }\end{array}$ \\
\hline Práctica 8 & Medio & $\begin{array}{l}\text { Empresarial (subvenciones, convenios, } \\
\text { capital privado) }\end{array}$ & $\begin{array}{l}\text { A mediano } \\
\text { plazo }\end{array}$ \\
\hline Práctica 9 & Medio & $\begin{array}{l}\text { Empresarial (subvenciones, convenios, } \\
\text { capital privado) }\end{array}$ & $\begin{array}{l}\text { A mediano } \\
\text { plazo }\end{array}$ \\
\hline
\end{tabular}

Fuente: elaboración propia

Todas estas experiencias se han referido al atractivo natural y al patrimonio construido como un recurso para promocionar la ocupación de estas áreas. La singularidad del paisaje de montaña en el Alto Aragón es uno de los factores que más se repite a la hora de explicar las razones para volver a ocupar los deshabitados. Ahora bien, mientras que las experiencias caracterizadas por la okupación y la vida alternativa se centran en el contacto permanente con la naturaleza y con los recursos que esta proporciona como factor atractor principal, en las experiencias significadas por el desarrollo turístico especializado estos valores se reconocen como recurso para promocionar su ocupación y garantizar cierta actividad comercial y económica. Conviene reseñar que el valor del medio natural es especialmente referido por aquellos que provienen de grandes ciudades del norte de Europa.

Por otro lado, todas las experiencias han intervenido, en mayor o menor medida, los bienes inmuebles existentes, fundamentalmente las construcciones aisladas destinadas al uso de vivienda, en la mayoría de casos para adaptarlas a las nuevas necesidades. Ahora bien, el tipo de obra y transformación ha sido muy distinto. Mientras que hay casos en los que únicamente se ha acondicionado el interior de los edificios y se han arreglado las carpinterías para que estas construcciones pudieran ser mínimamente habitables, también hay otros casos en los que se ha reformado de manera integral el pueblo, incorporando nuevos materiales, redefiniendo usos y adaptando su trazado a estos nuevos usos. Entre estos extremos también existen casos en los que se ha ampliado parcialmente el edificio y se ha alterado su fachada para disponer de huecos mayores o incluso miradores de gran superficie. Tan solo en las experiencias de intervención en deshabitados por parte de organismos y fundaciones de diversa índole se encuentran casos en los que se reconstruye el conjunto del deshabitado y se adecua a los nuevos usos, siempre vinculados al turismo (esquí, senderismo, camino de Santiago, etc.), al turismo de eventos (bodas, reuniones familiares, reuniones de empresa, etc.) o al turismo especializado (enoturismo, adaptado y accesible para personas con diversidad funcional, encuentros de yoga o ciclismo, entre otros). La mayoría de las veces, el proyecto se le encarga a un estudio de 
arquitectura, y en ese momento la herencia construida empieza a formar parte de una intervención de gran empaque y cuidado de detalle que muchas veces supera en extensión la propia superficie del deshabitado original.

Sea como fuere, resulta clave el hecho de que todavía no exista ningún desarrollo normativo al respecto más allá de que la única mención sea la realizada en la Ley de Turismo (Gobierno de Aragón, 2016), por la cual los pueblos recuperados deben respetar "los valores de la arquitectura tradicional de la zona" (art. 5I, num. 2); aquí se entiende como pueblo recuperado a aquel

núcleo deshabitado que se rehabilita y acondiciona para prestar una oferta turística de alojamiento en una o varias de sus modalidades, y que responde a un proyecto unitario de planificación, gestión y explotación empresarial, cumpliendo los requisitos establecidos reglamentariamente. (Art. 5I, num. I)

Por último, resulta destacable que uno de los retos principales radica en poder adaptarse a los desafíos de esta nueva vida sin posibilidad alguna de establecer lazos directos (por ser inexistentes) con las generaciones que una vez poblaron estos lugares. Las relaciones necesarias con las actividades económicas revelan hasta qué punto (además de las prácticas de movilidad y el uso de las infraestructuras de tecnología y comunicación) el medio natural y el patrimonio heredado son constitutivos de la vida cotidiana y de que, según el caso, se llegue a caracterizar la experiencia como mínimamente estable en el tiempo. Sin embargo, para las experiencias de intervención fundadas en el desarrollo turístico especializado, existe una reflexión profunda sobre el impacto que se quiere conseguir con estas prácticas. No debe perderse de vista, en primer lugar, que la gente no vive en esos "pueblos recuperados”, ya que la vida en comunidad únicamente "tiene lugar" en periodos de alta demanda, siguiendo criterios de ocupación y tarifas; en segundo lugar, tampoco hay que olvidar que las demandas de los nuevos usos que se instalan pueden acabar por degollar fácilmente la herencia construida, no solo de los valores de la arquitectura tradicional bien entendida, sino de la morfología y trama del núcleo primigenio sobre el que se está interviniendo.

\section{Conclusiones: patrones de cambio en el}

\section{territorio}

De la investigación realizada se desprende que no se puede defender la vida alternativa como una solución al fenómeno general de despoblación y, de manera concreta, de los deshabitados. Son muchos los que, en términos de cohesión social, dudan acerca de apostar por la recuperación de pueblos ya deshabitados cuando en la actualidad hay muchos que aún luchan por su supervivencia. Aun así, en términos de conservación del patrimonio, desarrollo cultural e innovación social, estas prácticas constituyen patrones de comportamiento y de cambio en el territorio cuyo valor debe ser reconocido.

La metodología de análisis etnográfica ha permitido descubrir los cambios realizados por estas comunidades en el territorio y las demandas básicas de estas para el reestablecimiento de una mínima masa crítica en un territorio desocupado. A través de la participación en las prácticas realizadas por los entrevistados y de la propia inmersión en la vida cotidiana experimentada, se ha podido desarrollar una comprensión de las dimensiones pragmáticas y atractivas que caracterizan la convivencia y la vida cotidiana de las comunidades que habitan estos espacios. Gracias a este estudio, se ha desarrollado un entendimiento sobre el papel que juegan los valores naturales -en diferente intensidad- en la elaboración y caracterización de cada una de las experiencias de ocupación de los entrevistados.

Pese a que el estado inicial de conservación de los diferentes deshabitados era dispar, en términos generales sí se cumple que su ocupación informal, discontinua y, en algún caso, al margen de lo establecido en los documentos de regulación jurídica por los que se regulan tales áreas constituye un mecanismo para preservar y mantener el 
entorno rural. Del mismo modo, parece razonable intervenir sobre lo construido siguiendo unos principios comunes que permitan garantizar el mantenimiento de los criterios de construcción y diseño propios de la arquitectura rural tradicional, aunque esta cuestión no siempre se cumple.

Este tipo de experiencias (nuevas ofertas, economía rural apoyada, revalorización de las vías de paseo, etc.) nace de las demandas de un sector de la población inserto en las lógicas de la sociedad del consumo y de la explosión del fenómeno urbano en el territorio. Hasta cierto punto, las expectativas de vida en los deshabitados incluyen desde el inicio incuestionables consideraciones con respecto a las prácticas de movilidad y uso de las infraestructuras de tecnología y comunicación. Incluso, la mayor parte de los nuevos pobladores han confiado en la disponibilidad de las nuevas tecnologías, por lo que no debe subestimarse el grado en que estas configuran las expectativas de vida allí. De hecho, es revelador que en ocasiones las TIC se conviertan en una fuente necesaria para alcanzar unas condiciones mínimas de bienestar, no solo en términos de acceso a productos básicos de consumo o de servicios, sino en términos de mantener unas redes de apoyo e intercambio razonablemente permanentes, aunque dispersas.

El trabajo aquí presentado proporciona una valiosa información sobre cuáles son las razones que animan a ocupar los deshabitados. El perfil de población es variado, si bien sus intereses son comunes en torno a un pretendido contacto con el entorno natural de cierto valor paisajístico. Este enfoque puede ser de utilidad como complemento a la mayoría de metodologías actuales fundadas en un enfoque ascendente en el que se implican los agentes locales con el objeto de fomentar la participación de la población (como en el proyecto-método Leader). Este estudio abre una línea de trabajo que considera nuevas variables a la hora de caracterizar estos entornos despoblados desde un punto de vista cualitativo. Futuros trabajos pueden ahondar en esta visión ampliando el número de casos de estudio considerados y el número de entrevistas realizadas. De su análisis comparativo, combinando metodologías cuantitativas y cualitativas, puede llegar a inferirse el tipo de acciones a establecer para mejorar tales ámbitos espaciales y, de manera específica, para preservar el patrimonio cultural y arquitectónico de las áreas rurales.

\section{Referencias}

Abrams, J. B., Gosnell, H., Gill, N. J., y Klepeis, P. J. (20I2). Re-creating the rural, reconstructing nature: An international literature review of the environmental implications of amenity migration. Conservation and Society, IO(3), 270-284. https://www.doi.org/10.4103/0972-4923.101837

Acín, J. L. (1994). Las otras lluvias. Pueblos deshabitados del Alto Aragón. Zaragoza: Ibercaja.

Acín, J. L. (1997). Paisajes con memoria. Viaje a los pueblos deshabitados del Alto Aragón. Zaragoza: Prames.

Acín, J. L., y Pinilla, V. (coords.). (1995). Pueblos abandonados. ¿Un mundo perdido? Zaragoza: Astral.

Aguilar, M. J. (2009). Despoblación y envejecimiento en el medio rural de Castilla La Mancha. Un análisis desde la perspectiva de género. En L. López, A. Abellán y D. Godenau (coords.), Despoblación, envejecimiento y territorio: un análisis sobre la población española (pp. 77-89). León: Universidad de León.

Aineto: una experiencia de repoblación en el Altoaragón. (1984). Trabajo presentado en el Congreso sobre Pueblos Deshabitados. Ministerio de Cultura, Dirección General de la Juventud y Promoción Sociocultural, Madrid.

Allanegui, G. (1979). Arquitectura popular de Aragón. Zaragoza: Librería General.

Allanegui, G., y Souto, M. (2003). Patrimonio etnológico en Aragón. Un presente con futuro. Trébede: Mensual Aragonés de Análisis, Opinión y Cultura, (72), 13-18.

Anaut-Bravo, S. (2009). Políticas sociales municipales contra el sobre-envejecimiento y la despoblación rural. La acción pública en Aibar (Navarra). En L. López, A. Abellán y D. Godenau (coords.), Despoblación, envejecimiento y territorio: un análisis sobre la población española (pp. 505-514). León: Universidad de León. 
Andrey, J. A. (2009). Implicaciones socioterritoriales de la despoblación en la montaña astur-galaico-leonesa. En L. López, A. Abellán y D. Godenau (coords.), Despoblación, envejecimiento y territorio: un análisis sobre la población española (pp. 547-566). León: Universidad de León.

Arango, J. (1976). Cambio económico y movimientos migratorios en la España Oriental del primer tercio del siglo XX: algunas hipótesis sobre determinantes y consecuencias. Hacienda Pública Española, (38), 5 I-80.

Ayuda, M. I., Pinilla, V. J., y Sáez, L. A. (2002). El problema de la despoblación en Aragón. Causas, características y perspectivas. Revista de Demografía Histórica, I8(I), I37-I73. Recuperado de https://www.dialnet.unirioja .es/servlet/articulo?codigo $=104079$

Ayuda, M. I., y Pinilla, V. J. (2002). El proceso de desertización demográfica de la montaña pirenaica en el largo plazo: Aragón. Ager, 2, 102-138. Recuperado de https://www.recyt.fecyt.es/index.php/AGER/article/view 17025

Benson, M. (20II). The movement beyond (lifestyle) migration: Mobile practices and the constitution of a better way of life. Mobilities, 6(2), 22I-235. https://www.doi.org/I0.1080/17450101.2011.55290I

Benson, M., y O'Reilly, K. (2009). Migration and the search for a better way of life: A critical exploration of lifestyle migration. The Sociological Review, 57(4), 608-625. https://www.doi.org/I0.IIII/j.I467-954X.2009.01864.x

Bernard-Rivera, P. M. (1992). Programa global para la rehabilitación de núcleos deshabitados y semideshabitados en áreas de interés especial del Pirineo. Zaragoza: DGA.

Bernard-Rivera, P. M., y Castellanos, J. M. (1982). Pueblos deshabitados del Alto Aragón. Estudio de la comarca de Sobrarbe. Zaragoza: Colegio Oficial de Arquitectos de Aragón.

Briet, L., y Acín, J. L. (2003). Bellezas del Alto Aragón. Huesca: Diputación de Huesca.

Caro-Baroja, J. (199I). Los pueblos de la península ibérica: temas de etnografía española. Barcelona: Crítica.

Castán, A. (2009). Lugares del Alto Aragón. Las comarcas pueblo a pueblo. Huesca: Publicaciones y Ediciones del Alto Aragón.

Castán, A., Calvera, E., e Iglesias, M. (1990). Huesca de la A a la Z. Huesca: Alto Aragón.

Chi, G., y Marcouiller, D. W. (2013). In-migration to remote rural regions: The relative impacts of natural amenities and land developability. Landscape and Urban Planning, II7, 22-31. https://www.doi.org/ı.ıоı/j.landurb plan.2013.04.012

Cloke, P. (1985). Counterurbanization: A rural perspective. Geography, 70(I), I3-23. Recuperado de https://www.r esearchgate.net/publication/280778430_Counterurbanisation_a_rural_perspective

Cloke, P. (1988). Policies and plans for rural people. An international perspective. Londres: Unwin Hyman.

Collantes, F. (200I). El declive demográfico de la montaña española, I860-199ı: revisión crítica de propuestas teóricas. Historia Agraria, (24), 203-225. Recuperado de https://www.repositori.uji.es/xmlui/handle/ı0234/ 130986

Comas-D’Argemir, D. (1995). Familia, sistemas de herencia y estratificación social. Estrategias hereditarias y despoblación. En J. L. Acín y V. Pinilla (coords.), Pueblos abandonados. ¿Un mundo perdido? (pp. I4I-I52). Zaragoza: Astral.

Dam, F. van, Heins, S., y Elbersen, B. S. (2002). Lay discourses of the rural and stated and revealed preferences for rural living. Some evidence of the existence of a rural idyll in the Netherlands. Journal of Rural Studies, I8(4), 46I-476. https://www.doi.org/I0.10I6/S०743-0167(02)00035-9

Díaz, J. (1995). Las aldeas perdidas. En J. L. Acín y V. Pinilla (coords.), Pueblos abandonados. ¿Un mundo perdido? (pp. 187-202). Zaragoza: Astral. 
Elshof, H., Haartsen, T., van Wissen, L., y Mulder, C. (2017). The influence of village attractiveness on flows of movers in a declining rural region. Journal of Rural Studies, 56, 39-52. https://www.doi.org/ı0.10I6/j.jrurst ud.2017.07.004

Feduchi, L. (1976). Itinerarios de la arquitectura popular española. Cataluña, Aragón, Baleares, Levante. Barcelona: Blume.

Flores, C. (1973). Arquitectura popular española. Madrid: Aguilar.

Gavín Lanzuela, M. L. (coord.). (2005). Catálogo de pueblos y municipios de Aragón. Estadística de población y nomenclaturas toponímicas entre 1900 y 2004. Zaragoza: Gobierno de Aragón, Servicios de Estudios. Recuperado de https://www.aragon.es/estaticos/ImportFiles/II/docs/Areas/Territor/Municip\%2oentidsi ngul/CatlgPuebl/CATALOGO_PUEBLOS.pdf

Germán, L. (1995). Crecimiento económico y disparidades espaciales. Notas para su estudio y aplicación a la industrialización española. En J. L. Acín y V. Pinilla (coords.), Pueblos abandonados. ¿Un mundo perdido? (pp. 19-34). Zaragoza: Astral.

Red Natural de Aragón. (2003-2007). Prames.

Gobierno de Aragón. (2005). Directrices Parciales de Ordenación Territorial del Pirineo Aragonés. Recuperado de https://www.aragon.es/DepartamentosOrganismosPublicos/Departamentos/VertebracionTerritorioM ovilidadVivienda/AreasTematicas/OrdenacionTerritorial/Directrices/ci.Copy-of-Directriceszonales.detal leDepartamento?channelSelected=83a536552883a210VgnVCMioooo0450ar5acRCRD

Gobierno de Aragón. (20II-2015). Departamento de Agricultura, Ganadería y Medio Ambiente. VIII legislatura. Recuperado de https://www.ecomisiones.cortesaragon.es/index.php?option=com_content\&view=article\&id $=2627 \% 3$ Aayudasriadas $\&$ catid $=233 \&$ Itemid $=25$

Gobierno de Aragón. (20I4). Estrategia de Ordenación Territorial de Aragón. Recuperado de https://www.aragon.es/estaticos/GobiernoAragon/Departamentos/PoliticaTerritorialInterior/Areas/o I_Ordenacion_territorio/EOTA/Proyecto_EOTA_DOCS_0320I4/MODELO\%20TERRITORIAL\%20$\% 20 \% 20$ ESTRATEGIAS\%20Y\%20NORMAS.pdf

Gobierno de Aragón. (2016). Decreto Legislativo I/20I6, de 26 de julio, del Gobierno de Aragón, por el que se aprueba el texto refundido de la Ley del Turismo de Aragón. Departamento de Vertebración del Territorio, Movilidad y Vivienda. Boletín Oficial de Aragón, (149). Recuperado de https://www.boa.aragon.es/cgi-bin /EBOA/BRSCGI?CMD=VEROBJ\&MLKOB=920376642323

Halliday, J., y Coombes, M. (1995). In search of counterurbanisation: Some evidence from Devon on the relationship between patterns of migration and motivation. Journal of Rural Studies, II(4), 433-446. https:/ /www.doi.org/10.1016/0743-0167(95)00032-I

Laglera, C. (2010). Despoblados en Huesca. Recuperado de https://www.despobladosenhuesca.com

Laglera, C. (20I4a). Despoblados de Huesca, tomo I: Ribagorza-Litera. Zaragoza: Pirineo.

Laglera, C. (20I4b). Despoblados de Huesca, tomo II: Jacetania-Alto Gállego-Hoya de Huesca. Zaragoza: Pirineo.

Laglera, C. (2015). Despoblados de Huesca, tomo III: Sobrarbe-Somontano. Zaragoza: Pirineo.

Laliena, A. C. (2004). El movimiento neo-rural en el Pirineo Aragonés. Un estudio de caso: la asociación Artiborain. Infomes 6. Recuperado de https://www.ceddar.org/content/files/articulof_265_02_Informes-20 04-4.pdf

Lardiés, R., y Castelló, A. (2015). Migraciones ligadas al estilo de vida en zonas rurales de Aragón. En I Congreso Internacional de Migraciones Contemporáneas, Territorio y Urbanismo: actas del congreso (pp. 243-258). 
Cartagena: Universidad Politécnica de Cartagena. Recuperado de https://www.repositorio.upct.es/handle $/ 10317 / 5112$

López, L., Abellán, A., y Godenau, D. (coords.). (2009). Despoblación, envejecimiento y territorio: un análisis sobre la población española. León: Universidad de León.

Marín-Gavín, S. (2013). El paisaje de la memoria: agrupación, análisis y valoración del conjunto de los pueblos deshabitados del Altoaragón: el planeamiento supramunicipal como clave para la regulación de su recuperación (Tesis de maestría). Universidad Politécnica de Cataluña, Barcelona. Recuperado de https:// www.upcommons.upc.edu/handle/2099.I/19973

Marín-Gavín, S. (2018). Pueblos recuperados en el Alto Aragón. Huesca: Diputación Provincial de Huesca.

Marín-Yaseli, M. L. (2006). Más de veinte años de políticas de desarrollo rural en el Pirineo aragonés. Ager: Revista de Estudios sobre Despoblación y Desarrollo Rural, (5), I27-154. Recuperado de https://www.recyt.fecyt.es/ index.php/AGER/article/view/7048/5588

Martínez-Fernández, C., Kubo, N., Noya, A., y Weyman, T. (20I2). Demographic change and local development: Shrinkage, regeneration and social dynamics. Local Economic and Employment Development (LEED)Organización para la Cooperación y el Desarrollo Económicos (OCDE). Working Paper Series. Recuperado de https://www.oecd-ilibrary.org/docserver/9789264I80468-en.pdf?expires=15432522I4\&id=id\&accname=gue st\&checksum=42EDB8EB699279530E94095 $\mathrm{A}_{419} \mathrm{D}_{3} \mathrm{EFF}$

Mitchell, C. J. A. (2004). Making sense of counterurbanization. Journal of Rural Studies, 20(I), 15-34. https://ww w.doi.org/10.1016/S0743-0167(03)00031-7

Naval-Mas, A. (1988). Arquitectura doméstica del Somontano en el Alto Aragón. Huesca: Cremallón.

Parlamento Europeo. (I.o de junio de 20I6). Zonas escasamente pobladas y severamente despobladas: un problema creciente en la UE. Aragón en Europa. Recuperado de https://www.aragoneneuropa.wordpress.com/2016/06 /10/zonas-escasamente-pobladas-y-severamente-despobladas-un-problema-creciente-en-la-ue-sparsely-popul ated-and-severely-depopulated-areas-a-growing-problem-in-the-eu/

Presidencia de las Cortes de Aragón. (2000). Plan Integral de Política Demográfica. Boletín Oficial de las Cortes de Aragón, (80), 3560-3574. Recuperado de https://www.bases.cortesaragon.es/bases/ndocumenVIII.nsf/e8 6600a24e054a6ici2576d2002e55ic/bif5589f70d6dceoci257ecboo2ef778/\$FILE/PLAN.pdf

Pujadas, J. J. (1995). Identidad cultural y despoblación. En J. L. Acín y V. Pinilla (coords.), Pueblos abandonados. ¿Un mundo perdido? (pp. 215-225). Zaragoza: Astral.

Ruiz, A. (2014). Planeamiento supramunicipal y construcción del territorio aragonés, España. Bitácora UrbanoTerrritorial, 2(24), 31-4I. https://www.doi.org/10.15446/bitacora

Sáez, L. A. (coord.). (20I6). Temas pendientes sobre despoblación. Curso de Verano de la Universidad de Teruel, del 4 al 6 de Julio. Universidad de Verano de Teruel.

Sáez, L. A., Ayuda, M. I., y Pinilla, V. (2008). Políticas inmigratorias frente a la despoblación: el caso de Aragón. En: XV Encuentro de Economía Pública: políticas públicas y migración. Salamanca. Recuperado de https: //www.dialnet.unirioja.es/servlet/articulo?codigo=3136560

Sáez, L. A., Pinilla, V., y Ayuda, M. I. (200I). Políticas ante la despoblación en el medio rural: un enfoque desde la demanda. Ager, I, 2II-232. Recuperado de https://www.recyt.fecyt.es/index.php/AGER/article/view/6990

Sánchez, M. E. (1995). Réquiem por un patrimonio olvidado: el etnográfico. En J. L. Acín y V. Pinilla (coords.), Pueblos abandonados. ¿Un mundo perdido? (pp. 167-176). Zaragoza: Astral.

Sanz, A. (2016). Discursos en torno a la despoblación en Teruel desde la prensa escrita. Ager, 20, 105-137. 
Satué, E. (1991). El Pirineo abandonado. Zaragoza: Diputación General de Aragón, Departamento de Cultura y Educación.

Serrano, C. (coord.). (2007). Despoblación y territorio. Zaragoza: Centro de Estudios sobre la Despoblación y Desarrollo de Áreas Rurales (Ceddar), Rolde de Estudios Aragonenses, Diputación Provincial de Zaragoza.

Silvestre, J. (2003). Migraciones interiores y mercado de trabajo en España (1877-1936) (Tesis doctoral). Universidad de Zaragoza, Zaragoza.

Slomp, H. J. (2004). La despoblación del medio rural español. Un estudio sobre las causas, consecuencias y política con respecto a un proceso de éxodo. Diversificación rural en Matarraña, Teruel (Tesis doctoral). Universidad de Groninga, Países Bajos.

Ubieto, A. (1984). Historia de Aragón, los pueblos y los despoblados. Zaragoza: Anubar.

VVAA. (s. f.). Estudio monográfico. Lúsera, Huesca, trabajo realizado por el grupo scout San Viator. Trabajo finalista en Temas monográficos aragoneses, Peña Solera Aragonesa de Zaragoza.

\section{Notas}

* Artículo de investigación

Licencia Creative Commons CC BY-4.o 